\title{
Integration of Economic Information with Design and Manufacturing Systems
}

Aydan Kutay and Susan Finger

CMU-RI-TR-91-02

The Robotics Institute

Carnegie Mellon University

Pittsburgh, Pennsylvania 15213

Manch 1991

사 1991 Carnegie Mellon University 


\begin{abstract}
Most of the life-cycle cost of a product is determined by the decisions made during the preliminary product design stage. As competition causes a reduction in product lead times, an increase in demands for product quality, and an increase in the need for flexibility in product differentiation, the need for better cost estimation tools in the design stage also increases. During preliminary design, the cost implications of design decisions may not be computable with precision, but the information needed to make better informed cost tradeoffs can be made available to the designer. In this paper, we present a methodology to compute the costs of product features for each of the stages in a product's life-cycle. We propose to integrate this methodology with an existing design system in order to give rapid feedback to the designer on the costs attributable to particular design features. We use these costs to provide economic feedback to the designer on the feasibility and economics of manufacture, assembly, testing, marketing, etc.
\end{abstract}




\section{Introduction}

Increasingly, American industry needs to reduce its cost of production -- from design through delivery. Cost reduction can be achieved both through technology and through advanced management techniques. Despite improvements in technology and management science, low cost manufacturing is still driven by product design [Hayes 88 ]. Many studies have shown that as much as $80-85 \%$ of the manufacturing costs of a product are directly attributable to decisions made during the preliminary product design stage [Brei 88], [Whitney 88]. A cost estimation tool that gives rapid feedback to the designer on product costs corresponding to a particular set of design attributes is necessary to reduce overall manufacturing costs. The designer can drive costs up through complex part design, excessively tight dimensional tolerances, unnecessarily close surface finish specifications, and improper material selection. Linking the final product costs to features of the design will increase the designer's ability to reduce costs as well as to learn about the production system.

In this paper we present our research on the development of methodologies to integrate economic information with product design and manufacturing so that economic feedback on the feasibility and economics of manufacture, assembly, testing, etc. can be rapidly provided to the designer. Our goal is to develop a methodology for the study of the economics of new technologies, particularly the issue of integrating economic, business, marketing information with the design and manufacturing system.

In [Kutay 89], we presented a methodology to assess the strategic benefits from new technologies that accrue from increased flexibility and reduced response times. In this paper our focus is the development of a methodology for the economic analysis of design features. We first present a methodology to integrate cost into the design system so that a designer can receive feedback on the economic implications of design decisions as they are made.

A designer must be able to relate reliability and quality both to cost and to marketability [Byme 86], [Taguchi 78], [Taguchi 86]. The methodology presented in this paper is built on a framework that gives the designer the ability to make design decisions based on feedback from many different perspectives, that is, from different stages in the design's life-cycle. The framework integrates the perspectives that affect product design by defining a common view of information within and in between the system perspectives. It also provides the designer with the information necessary to decide which technology to use to manufacture the product and to design the part given the limitations of the selected technology.

Our goal is to provide the link between design features of a product and the life-cycle costs from conceptual design to the point of disposal. The development of the entire methodology is beyond the scope of this paper. Here, we address the method to track economic and cost information through a product's life-cycle.

\section{Integrated Product Design System}

To integrate cost information into the design system will require an economic analysis of features and the interactions of features from each perspective. Our system will enable the designer to link design decisions to cost drivers. In firms that produce a wide variety of products with a large range of complexity, the designer may be unaware of the relationship between design decisions and product costs. The integrated product design system offers the designer the ability to relate design decisions to product costs.

The product design system we are developing can accumulate and manage information associated with the shared design representation. The conceptual framework of the system is illustrated in Figure 1. As shown in the figure, there are two types of information flows in product design: the left-hand side is the product oriented information and the right-hand side is the business/economics oriented information. The two types of information flows feed into each other through the following types of interactions:

- Initial product specifications are determined based on market analysis and the strategic goals 
of the firm in the market.

- A design is generated that meets the final specifications for the product and that is feasible to manufacture, assemble, test, service based on the information available in the design stage.

- A detailed manufacturing plan is created given the constraints imposed by the technologies available to the firm.

- The product design is refined based on the capabilities of the available technology. These refinements are fed back to product design.

- Products are manufactured, inspected, assembled, tested, and shipped. Knowledge gained in each stage is fed back to previous stages.

- For each product, the realized costs in each stage of production, from manufacturing to service, are allocated to cost drivers by the accounting perspective.

- The goals in manufacturing planning and strategic planning are re-evaluated given the accounting information that is generated. These revisions are fed back to product design.

Our approach is based on a broader view of manufacturing than current CAD systems which view manufacturing as the set of activities to transform engineering documentation into physical artifacts. In our integrated methodology, as illustrated in Figure 1, product design plays the central role within the broader scope of manufacturing so that the designer can integrate life-cycle product cost information into the design decision processes. Information generated in the accounting perspective, such as inventory costs, labor costs, variable costs, and sales revenue, influence mamufacturing planning, and strategic planning perspectives and therefore influence product specifications to feed into the design stage once again. The structure of interactions between costs, sales revenue, inventory costs reduction, and the strategic position of the firm in the market is determined based on the framework explained in detail in [Kutay 89].

\section{Concurrent Product Design}

In traditional design and manufacturing systems, it is difficult for designers to consider the impiications of their decisions for manufacturing, process planning, and inspectability or other life-cycle issues such as serviceability and disposability. This difficulty arises from many causes; often the necessary information is unavailable or incomplete or too detailed to be useful. In addition, many analytical models can be applied only after the design is complete, that is, after all the important design decisions and commitments have been made. These difficulties have led to many less than optimal designs when the entire life of a product, from conception to disposal, is considered.

The existing methods to estimate product costs vary widely depending on the stage of design and production [Ulrich 90]. When the product is at the conceptual stage, product costs are estimated based on historical data obtained from similar products produced with a similar technology. At the layout stage, cost data from products with similar components are used for estimation. The refinement of cost estimates becomes possible only when the product reaches the detailed drawings stage. The designer's access to cost information is fragmentary and incomplete throughout this process. Actual product costs cannot be obtained until production starts. Inconsistencies in information flow and lack of access to complete information can be observed in current CAD systems. For example, current design checkers work only after the design is completed. To avoid unnecessary iterations in the design stage, critiques of incomplete designs are necessary.

In the design system which has been implemented [Finger 91], experts give feedback to a designer only on the feasibility or desirability of the features of the design. This advice may be contradictory; for example, the assembly perspective may criticize the design choice of using screws instead of a snap fit, 


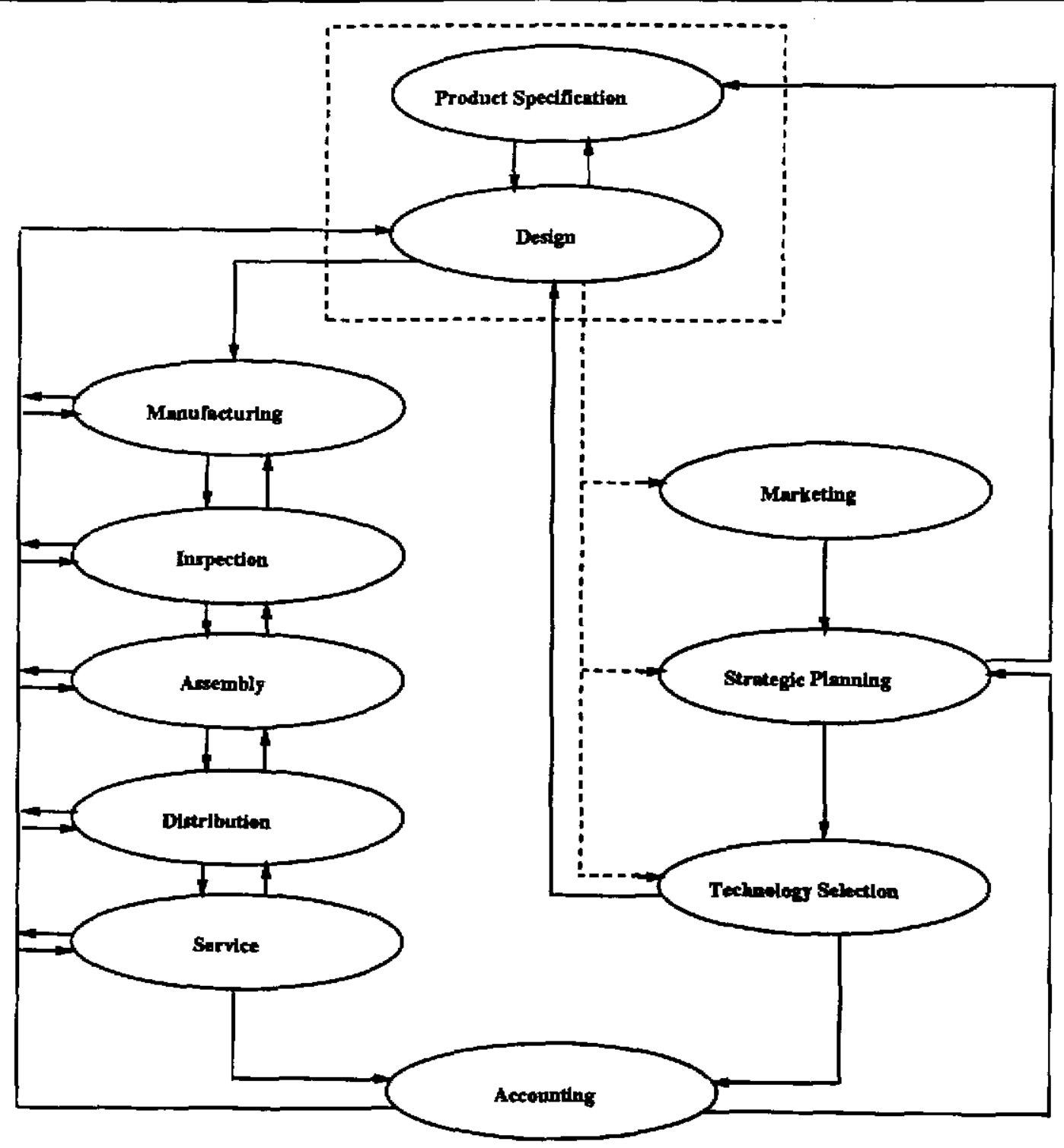

Figure 1: The Integrated Product Design System

while the service perspective would have the opposite preferences. The designer must be able to make informed tradeoffs among conflicting constraints. Currently, a designer does not have enough information to weigh the cost implications of this decision over the life of the product.

In addition, a design may be manufacturable using one process, but infeasible using an altemative process. Thus, two parts can have different manufacturing processes and different forms, but still fulfill the same behavioral requirements. Because current CAD systems are driven by form, exploring altemative manufacturing processes requires complete redesign of the part. Our approach to resolving the problems of inconsistent and incomplete information flow to the designer is to create a system in which a designer can compose a design using high-level entities, called features, that have behavioral and geometrical attributes. As the design evolves, the designer receives feedback from different perspectives representing the concerns of manufacturing, analysis, inspection, maintenance, etc. 
One motivation for creating feature-based design systems is that a product design is viewed differently as the design evolves and as viewed by different experts, such as the machining expert, the molding expert, or the inspection expert. The attributes of the design, that is, the features, that add to costs are different for each perspective. If expertise is to be transmitted automatically to the designer, it is essential that each expert be able to interpret the design and generate comments about the design using the important features in that perspective.

A central idea in the integrated design system is that the shared representation of the product design contains only the information needed by more than one perspective. For example, the marketing information on a product will be contained within the marketing perspective. The interaction between the perspectives occurs through the blackboand architecture. The shared, neutral representation is stored on the blackboard. Each perspective can interpret and reference information in the shared model. Each perspective can create construction rules to describe how its own information can be obtained, structured, and computed from the shared model [Finger 90], [Finger 91].

\section{Integration of Life-Cycle Costing into Design Systems}

A critical part of the integrated product design system depicted in Figure 1 is the interaction among the technology of the firm and the design and the manufacturing of its products. In the manufacturing planning stage, methods for realizing the designed shapes are determined based upon the constraints imposed by available technology of the firm under the criterion of minimum production cost. Product design configurations are then re-evaluated if the product design cannot meet the manufacturing criteria or other constraints imposed by the available technology. Requirements for the product performance at the product design stage and those for the product manufacturing cost at the manufacturing planning stage may have conflicting relationships. We synthesize the evaluations made at the product design stage and manufacturing planning stage to attain a better design solution, rather than attaining individual optima in each stage through sequential decision making.

\subsection{Linking Component Costs to Design Features}

High productivity and quality stem from the thoughtful design of products and manufacturing systems. Several studies have shown that $80 \%$ of a product's life-cycle cost is fixed early in design and that at least $80 \%$ of quality defects can be traced to flaws in design. In this section we present a methodology to track costs to particular components of a product. This methodology is a necessary prerequisite to communicate production cost information to product designers to enable the rapid production of highquality, inexpensive products.

Tracking the costs of design features requires precise identification of cost centers within the manufacturing organization and throughout the product's life cycle. Once the cost centers are specified, the activity measures that cause cost variation to occur must be determined. Therefore, each cost center must have a clear definition of its boundaries and of the time needed to create units of output.

Traditional product costing allocates all factory costs to products for the purpose of valuing inventory and the costs of goods sold. This approach results in cost systems that allocate costs based on direct labor and direct costs. However, in recent years, an important method of gaining competitive advantage has been to increase product and process complexity. Corporations have tended to increase the number of features and to design more complex products because price and market share advantages are perceived to outweigh the additional costs of managing the manufacturability of complex products [Cooper 89].

The manufacture of complex products often requires more supervision, quality control, inspection, machine and tool maintenance, and production control. In most accounting systems, these costs are classified as overhead costs. Management of overhead costs is becoming increasingly important with increased automation, shorter product life cycles, increased product differentiation, and faster customer 
response. The overhead component of manufacturing costs in many plants exceeds $50 \%$, with material costs accounting for $40 \%$ and direct labor only 10\% [Banker 89]. Existing cost accounting systems concentrate on direct labor and materials and hide the costs of overhead departments by simplistic allocations based on labor and material. Because overhead costs are allocated to products based on direct labor costs, the cost of products with more complex design features tends to be underestimated [Banker 89]. Therefore, new product costing methods are needed to understand the effect of design features on product costs. Because most of the overhead costs are committed during the design stage, a designer can significantly reduce manufacturing costs by making informed design decisions.

Linking product costs to design features can be achieved by identifying the cost drivers and investigating the demands placed on overhead departments. The primary cost drivers in the production system are transactions involving exchange of materials or exchange of information [Miller 85]. Based on Miller's work, we can group these transactions as:

- Logistical transactions: to order, execute, and confirm materials movement. Personnel involved with logistical transactions include indirect shopfloor workers, receiving, expediting, shipping, data entry, EDP, and accounting personnel.

- Balancing transactions: to match the supply of material, labor, and machines with demand. Personnel charged with balancing transactions include purchasing, materials planning, production control, forecasting, and scheduling workers.

- Quality transactions: to validate that production conforms to specifications. People in quality control, indirect engineering, and procurement perform quality transactions.

- Change transactions: to update manufacturing information. Manufacturing, industrial, and quality engineers involved with ECOs, schedules, routings, standards, specifications, and bills of materials perform change transactions.

These four group of transactions include only factory transactions. While manufacturing costs are important for tracking costs to products and eventually to design features, they are only a portion of the total costs of producing a product and delivering it to a customer. Many product costs are incurred below the gross margin line, particularly marketing, distribution, and service expenses [Johnson 88]. The costs of using different distribution channels or the costs of selling to different types of buyers must be traced to product design features for an accurate analysis. For example, if a product is sold with a maintenance agreement, it may be necessary to make tradeoffs between reducing manufacturing costs and reducing disassembly costs. Tracking product costs to design features based solely on factory costs would provide inaccurate estimates of the total cost of the product.

Tracking the selling, distribution, and service costs to design features is not an easy task. Because existing cost accounting practice is based on a line-item financial income statement and combines costs incurred outside the factory into aggregate functional categories, cost tracing of design features by making analogies to similar products is not a viable approach. To begin the process of tracing costs, estimates are needed of how marketing and sales time and effort are allocated across buyer segments and distribution channels for products with differing features. This will give a rough estimate of the cost drivers of marketing, sales, and distribution efforts so that analogies to products with similar features can be drawn when a new product design is evaluated for cost analysis.

Tracking the product costs to design features also requires an understanding of the sources of total process costs. Tracking the costs to processes can be accomplished at the level of the organization where the process occurs. First the organizational unit, or the cost center, must be identified. Then, the activity measures which cause cost variations to occur are determined. For the least automated process cost centers, the activity measure will be direct labor hours. Machine hours will be more relevant for automated process centers. Similary, the number of orders processed by the receiving department, the number of orders shipped by the shipping department, and the number of set-ups or pounds of material 
moved for an indirect labor department would be relevant measures of activity that cause cost variation to occur. The term of cost variation is the time period during which effective process control occurs [Johnson 88].

To make tradeoffs, designers need to know the costs of acquiring resources, material, capital, people in addition to knowing the cost of transforming these resources into final products and services, the costs of delivering a product with particular design features to customers, and the costs of servicing those products. A design system should accumulate costs, by product and its features, so that the designer will have a good understanding of the interaction between design features and the costs of producing a product with particular features. The lack of such an understanding will lead the designer to make critical design decisions about the complexity of design features based on a lack of information or based on averaged costs that incorporate cross subsidies and distortions. These cost distortions may eventually cause a company to become vulnerable to the strategies of competitors who understand the relationship between design features and life-cycle costs.

Thus, a design system must be able to track both overhead and direct costs to product design. If designers have a good understanding of the source of overhead costs, they can reduce these costs by modifying their design so that it meets the functional requirements but has a lower life-cycle cost.

For most production processes the product does not appear until the final assembly stage. Therefore, in our system, costs ane traced to components by estimating the demands of the component on both infactory and out-of-factory cost drivers. As suggested in [Johnson 88] and [Miller 85], the processes applied to each component are identified and process costs are estimated. Then, an estimate of the total number of transactions of each type are summed across all components to identify the total costs that are driven by each type of transaction. Once the total costs are identified, the cost per transaction of each type is calculated by simple division. Thus, cost per unit of driver is obtained, such as cost per inspection, cost per labor hour in a particular department, and cost per set-up. The outcome of this procedure is a set of cost centers each of which has a cost per unit of cost driver as in Table 1.

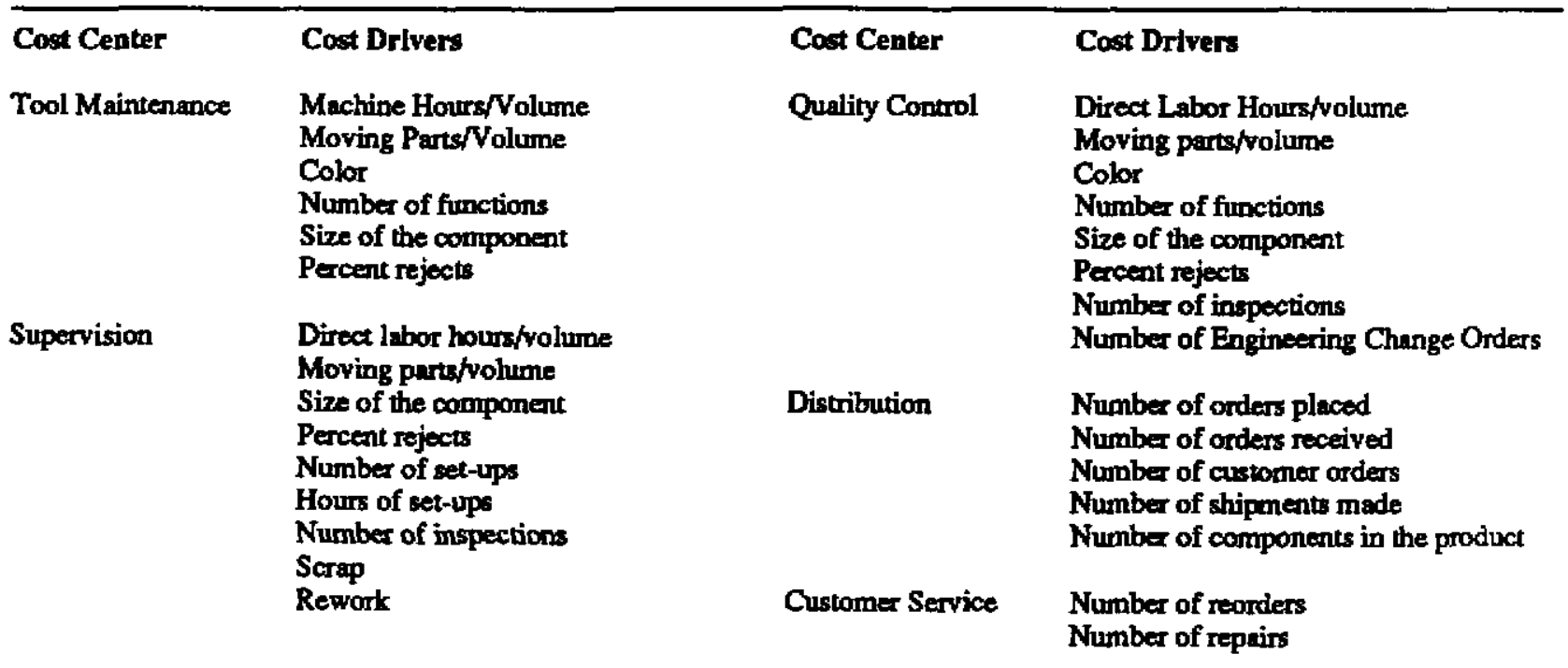

Table 1: Cost Centers and Drivers 


\subsection{Computing Component Costs}

Costs for each component can be estimated by finding the cost drivers used by that component in a particular cost center. These are then multiplied by the unit cost of each driver and summed to arrive at the fully traced cost for each component. Once costs for each component are identified, product costs are obtained by summing all component costs of the product. The process of estimating component costs can be expressed as:

where

$$
C_{k}=\sum_{i=1}^{I} \sum_{j=1}^{J} c_{i j} n_{i j k}
$$

$C_{k}=$ total cost of component $k$

$c_{i j}=$ cost per cost driver $j$ in cost center $i$

$n_{i j k}=$ number of times cost driver $j$ in cost center $i$ is used by the component $k$

Linking component costs to design features can be accomplished by analyzing the cost of components at a particular cost center as a function of the design features of the components. A regression model will be built to predict the contribution of particular design features to total cost drivers in a particular cost center. Since we know the cost per cost driver, the estimate of cost per design feature in each cost center can be obtained. The analysis can be formally explained as:

$$
C_{k i}=\sum_{i=1}^{L} f_{i i}\left(P_{l k i}\right)
$$

where

$$
\begin{aligned}
& C_{k i}=\text { cost of component } k \text { in cost center } i \\
& P_{l k i}=\text { vector of parameters for feature } l \text { in component } k \text { in cost center } i \\
& f_{i l}=\text { cost function for feature } l \text { in cost center } i
\end{aligned}
$$

This analysis will give us three types of information critical in the design of a product: total product costs per cost center and component, feature costs per cost center, and the contribution of feature parameters to feature costs in cost centers.

The estimates of feature costs will be based upon unit feature costs. Therefore, heteroscedasticity problems should not significantly affect the estimates. The Goldfeld-Quandt [1972] and White [1980] tests will be utilized to prevent any remaining problem of heteroscedasticity in the estimates. Multicollinearity tests including Belsey, Kuh and Welsch [1980] condition indices and eigen vector-based diagnostics will be utilized to obtain consistent estimates of feature costs.

In the feature-based design system described in Section 3, each perspective already has defined sets of features that can be extracted from the design to provide feedback and analysis to the designer. With the methodology that we have laid out here, cost functions can be assigned to the features so that in addition to providing information on the feasibility of a design, the perspectives can also provide information so that cost tradoffs can be performed.

\section{Future Work}

The major research question to be addressed is the construction of the design rules that include economic information. We plan to apply this methodology to two experimental manufacturing systems to discover how the costs relevant to designers can be obtained, stnuctured, and computed. We will enhance the existing design system to incorporate cost data with the manufacturing features and evaluate the extent to which our approach is viable within the context of the design and manufacturing of building products. We will also explore the generality of the model for firms operating in different markets with different product requirements. We will begin to define the framework in which global economic tradeoffs can be 
made between differing requirements in the life-cycle of a product. An ongoing element of our research is the continuous feedback between the development of integrated product design-manufacturing system and the strategic assessment model developed in [Kutay 89]. 


\section{References}

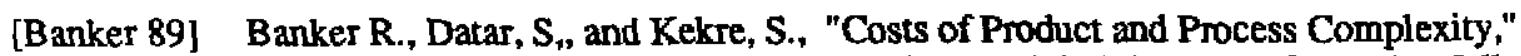
Technical Report, Graduate School of Industrial Administration, Camegie Mellon University, Winter 1989.

[Brei 88] Brei, M. L., Cralley, W. E., Dierolf, D., Owen, D. J., Richter, K. J. and Rogen, E., "Architecture and Integration Requirements for an ULCE Design Environment," Paper P-2063, Institute for Defense Analysis, April 1988.

[Byme 86] Byme, D. M. and Taguchi, S. , "The Taguchi Approach to Parameter Design," ASQC Quality Congress Transactions, American Society of Quality Control, Anaheim, CA, 1986.

[Cooper 89] Cooper, R., "Activity-Based Accounting System for Manufacturing Organizations," Harvard Conference on Accounting, Harvard University, January 1989.

[Finger 90] Finger, S. and Safier, S. A., "Representing and Recognizing Features in Mechanical Designs," Second International Conference on Design Theory and Methodology, DTM '90, ASME, Chicago, September, 1990, pp. 19-25.

[Finger 91] Finger, S., Fox, M. S., Prinz, F. B., and Rinderle, J. R., "Concurrent Design," Applied Artificial Intelligence, Vol. to appear, No. , 1991.

[Hayes 88] Hayes, R. H., Wheelwright, S. C. and Clark, K., Dynamic Manufacturing: Creating the Learning Organization, The Free Press, New York, 1988.

[Johnson 88] Johnson, H. T., "Activity-based Information: A Blueprint for World Class Accounting," Management Accounting, June 1988, pp. 23-30.

[Kutay 89] Kutay, A., "Assessment of the Economic Benefits of Automated Design and Prototyping," Technical Report, Robotics Institute, Canegie Mellon University, December 1989.

[Miller 85] Miller, J. G. and Vollmann, T. E., "The Hidden Factory," Harvard Business Review, September/October 1985, pp. 143-146.

[Taguchi 78] Taguchi, G., "Off-Line and On-Line Quality Control Systems," International Conference on Quality Control, Tokyo, 1978.

[Taguchi 86] Taguchi, G., Introduction to Quality Engineering, Asian Productivity Organization, (available from UNIPUB, New York), 1986.

[Ulrich 90] Ulich, K. T. and Fine, C. H., "Cost Estimation Tools to Support Product Design," ASME Manufacturing International Conference, American Society of Mechanical Engineers, March 1990.

[Whitney 88] Whitney, D. E., Nevins, J. L., DeFazio, T. L., Gustavson, R. E., Metzinger, R. W., Rourke, J. M. and Seltzer, D. S.. "The Strategic Approach to Product Design," Design and Analysis of Integrated Manufacturing Systems, National Academy Press, Washington, DC, 1988, pp. $200-223$. 